University of Nebraska - Lincoln

DigitalCommons@University of Nebraska - Lincoln

Faculty Publications from the Department of Engineering Mechanics

Mechanical \& Materials Engineering,

Department of

8-5-2005

\title{
Electrohydrodynamic instability of thin conductive liquid films
}

Xiangfa Wu

Department of Engineering Mechanics, University of Nebraska-Lincoln, xfwu@unlserve.unl.edu

Yuris A. Dzenis

Department of Engineering Mechanics, University of Nebraska-Lincoln, ydzenis@unl.edu

Follow this and additional works at: https://digitalcommons.unl.edu/engineeringmechanicsfacpub

Part of the Mechanical Engineering Commons

Wu, Xiangfa and Dzenis, Yuris A., "Electrohydrodynamic instability of thin conductive liquid films" (2005). Faculty Publications from the Department of Engineering Mechanics. 23.

https://digitalcommons.unl.edu/engineeringmechanicsfacpub/23

This Article is brought to you for free and open access by the Mechanical \& Materials Engineering, Department of at DigitalCommons@University of Nebraska - Lincoln. It has been accepted for inclusion in Faculty Publications from the Department of Engineering Mechanics by an authorized administrator of DigitalCommons@University of Nebraska - Lincoln. 
Published in Journal of Physics D: Applied Physics 38 (2005), pp. 2848-2850; doi 10.1088/0022-3727/38/16/017

Copyright (C) 2005 Institute of Physics Publishing Ltd. Used by permission. http://stacks.iop.org/JPhysD/38/2848

Submitted April 5, 2005; revised May 31, 2005; published August 5, 2005.

\title{
Electrohydrodynamic instability of thin conductive liquid films
}

\author{
Xiang-Fa Wu and Yuris A Dzenis \\ Department of Engineering Mechanics, Center for Materials Research and Analysis, \\ University of Nebraska-Lincoln, Lincoln, NE 68588-0526, USA
}

Corresponding author - X.-F. Wu, email xfwu@unlserve.unl.edu

\begin{abstract}
This paper considers the effect of surface charges on the surface instability of thin conductive liquid films. A characteristic relation is obtained for determining the wave number of the fastest growing mode as a function of surface tension, dispersive van der Waals force, and electrostatic tractions exerted by the film surface charges. Two natural length scales of the microsystem are further introduced to account for the coupling effects on the dewetting pattern development. The present results can be used for controlled surface pattern modulation in a spinodal-dewetting scenario via amplification of surface waves of selected modes.
\end{abstract}

\section{Introduction}

Surfaces of thin liquid films are morphologically unstable under the competing action of surface tension and dispersive van der Waals force between films and substrates [1]. In recent years, this phenomenon has attracted considerable attention due to its fundamental importance in understanding the mechanisms of surface pattern development and relevant modulation during dewetting of thin liquid and polymer films [2-12]. So far, most studies in this field have focused on the forces mentioned above and assumed that other possible instability-driving forces are negligible. New experimental and theoretical investigations have indicated that charged surfactants [13], external electric field [14-18], surface elasticity, and thermal field [19-24] may play a vital role in these surface phenomena. Among these, external electric field has been proposed for use in electrostatic lithography and surface pattern development due to the high sensitivity of thin liquid films to electrostatic forces $[15,16]$. It is expected that external electric field may lead to substantial reduction of the characteristic wavelengths of instabilities, thus overcoming the fundamental technological barrier to the production of increasingly small features such as the ones needed for integrated circuits [15]. In the latter investigations, the linear perturbation analysis of the electrically induced microstructure formation and pattern modulation in thin dielectric films indicated that a universal relation exists between the pattern wavelength and applied electric field such that

$$
\frac{\lambda}{\lambda_{0}}=2 \pi\left(\frac{E_{\mathrm{p}}}{E_{0}}\right)^{-3 / 2},
$$

where $\lambda_{0}=\varepsilon_{0} \varepsilon_{\mathrm{p}}\left(\varepsilon_{\mathrm{p}}-1\right) U^{2} / \gamma$ is a characteristic length corresponding to the relative strength of the electrostatic and Laplace pressure, $E_{0}=U / \lambda_{0}$ is a constant measuring the strength of electric field, and $\varepsilon_{0^{\prime}} \varepsilon_{\mathrm{p}^{\prime}}, U$, and $\gamma$ are the dielectric constant of vacuum, relative dielectric constant of the film, applied external voltage, and surface tension, respectively.

Clearly, relation (1) does not hold for conductive films such as conductive liquid and polymer films since there are no electric fields inside the conductive films. In this case, the negative Laplace pressure acting on the film surface is determined according to the Maxwell stress tensor on the charged surface [25]. Therefore, in this paper, we consider the effect of surface charges on the surface instability of thin conductive liquid films. Based on linear perturbation of electrohydrodynamics of a thin liquid film, a novel characteristic relation is derived for determining the wave number of the fastest growing mode as a function of surface tension, dispersive van der Waals force, and electrostatic tractions exerted by the film surface charges. Two natural length scales of the microsystem are introduced to account for the coupling effects on the dewetting pattern development. The relevant characteristic curves are plotted under conditions of various film thickness and surface charges. 


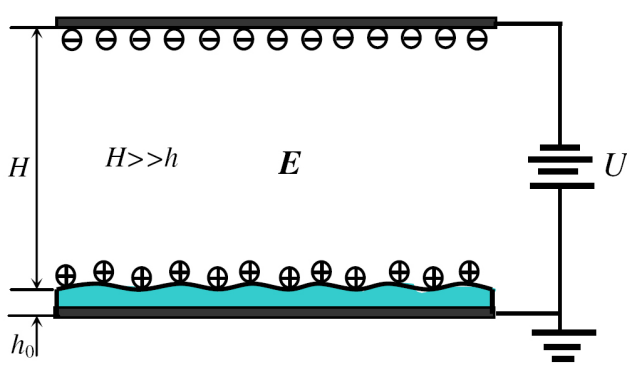

Figure 1. The system under consideration: a conductive liquid film on a solid conductor.

\section{Problem formulation and solution procedure}

Hereafter, we consider the electrohydrodynamic instability of a thin conductive liquid film of thickness $h_{0}$ on a flat conductive substrate, as shown in Figure 1. Similar to the parallel-plate capacitor, another flat conductive plate parallel to the substrate is introduced at a distance $H(H \gg h)$ to induce a uniform far electric field $E_{0}=U /\left(\varepsilon_{0} H\right)$. The surface charge density of unperturbed flat film is determined as $\sigma_{0}=E_{0} /(4 \pi)$. The thin liquid film may lose its surface stability under the combined action of surface tension, van der Waals forces, and electrostatic surface force. The overall pressure at the film surface with small perturbation may be expressed as

$$
p=p_{0}-\gamma \frac{\partial^{2} h}{\partial^{2} x}+p_{\mathrm{v}}(h)+p_{\mathrm{e}}(h),
$$

where $p_{0}$ is the atmospheric pressure; the second term is the Laplace pressure, stemming from the surface tension, $\gamma$ is the surface tension of the conductive film; the third term is the disjoining pressure at the film surface, i.e., $p_{\mathrm{v}}$ $=A /\left(6 \pi h^{3}\right)$ with $A$ being the Hamaker constant describing van der Waals interaction of the film with the substrate; and the last term is the negative electrostatic pressure. For unperturbed surface, the potential above the film surface may be expressed as $\phi=-4 \pi \sigma_{0} z$ with $z$ the vertical coordinate beginning from the flat film surface. For film surface with small perturbation

$$
h=h_{0}+\delta \cos (k x) \exp (t / \tau),
$$

with $\delta$ the perturbation amplitude, $k$ is the wave number and $\tau$ is the growth time scale, the potential above the film may be expressed as $\phi=-4 \pi \sigma_{0} z+\phi_{1}$ [25]. Here $\phi_{1}$ is a small correction, which satisfies the equation $\Delta \phi_{1}=0$ and vanishes for $z \rightarrow \infty$, which can be further expressed as $\phi_{1}=4 \pi \sigma_{0} \delta \cos (k x) \exp (-k z+t / \tau)$. On the film surface itself, the electrostatic potential must be a constant zero due to grounding as shown in Figure 1; thus the corresponding $\phi_{1}$ may be expressed as

$$
\phi_{1}=4 \pi \sigma_{0} \delta \cos (k x) \exp (t / \tau) \text { for } z=0 .
$$

The first order extension of the negative electrostatic surface pressure can be determined such that

$$
\begin{aligned}
p_{\mathrm{e}}(h) & =-\frac{E^{2}}{8 \pi} \approx-2 \pi \sigma_{0}^{2}-\left[k \sigma_{0} \phi_{1}\right]_{z=0} \\
& =-2 \pi \sigma_{0}^{2}-4 \pi \sigma_{0}^{2} k\left(h-h_{0}\right) .
\end{aligned}
$$

Furthermore, the modulation of $h$ gives rise to a lateral pressure gradient inside the film, inducing a Poiseuille flow $j$ as

$$
j=\frac{h^{3}}{3 \eta}\left(-\frac{\partial p}{\partial x}\right)
$$

where $\eta$ is the kinematic viscosity of the liquid film. A continuity equation enforces the mass conservation of the incompressible flow:

$$
\frac{\partial j}{\partial x}+\frac{\partial h}{\partial t}=0
$$

With the aid of (6), equation (7) reduces to

$$
\frac{\partial h}{\partial t}=\frac{h^{3}}{3 \eta} \frac{\partial^{2} p}{\partial^{2} x} .
$$

Substitution of (2) into (8) yields

$$
\frac{\partial h}{\partial t}=\frac{h^{3}}{3 \eta}\left[-\gamma \frac{\partial^{4} h}{\partial x^{4}}-\frac{\partial^{2} p_{\mathrm{e}}(h)}{\partial x^{2}}-\frac{A}{2 \pi h^{4}} \frac{\partial^{2} h}{\partial x^{2}}\right],
$$

where the higher order term $(\partial h / \partial x)^{2}$ is neglected. Now, consider the evolution of the film surface near $h_{0}$. Under small surface perturbation (3), the negative electrostatic surface pressure can be approximated using relation (5), thus equation (9) may be recast near $h=h_{0}$ such that

$$
\frac{\partial \xi}{\partial t}=\frac{h_{0}^{3}}{3 \eta}\left[-\gamma \frac{\partial^{4} \xi}{\partial x^{4}}-\left(4 \pi \sigma_{0}^{2} k+\frac{A}{2 \pi h_{0}^{4}}\right) \frac{\partial^{2} \xi}{\partial x^{2}}\right],
$$

where $\xi$ is the film surface perturbation such that $\xi=h-h_{0}$. Considering relation (3), one obtains the growth rate of the mode with wave number $k$ as

$$
\tau^{-1}=\frac{h_{0}^{3}}{3 \eta}\left(\frac{A k^{2}}{2 \pi h_{0}^{4}}+4 \pi \sigma_{0}^{2} k^{3}-\gamma k^{4}\right)
$$

from which the nontrivial wave number, $k_{\text {max }}$ with the fastest growth rate can be determined immediately as

$$
k_{\max }=\frac{\sqrt{\left(2 \pi \sigma_{0}^{2}\right)^{2}+A \gamma /\left(2 \pi h_{0}^{4}\right)}+2 \pi \sigma_{0}^{2}}{\gamma}
$$

or

$$
k_{\max }=\frac{\sqrt{E_{0}^{4}+32 \pi A \gamma / h_{0}^{4}}+E_{0}^{2}}{8 \pi \gamma} .
$$

Therefore, the undulated dewetting pattern will evolve with a clearly visible characteristic wavelength of $2 \pi / k_{\max }$. For zero voltage, the classic result for spinodal dewetting is obtained as $k_{\max }=[A /(2 \pi \gamma)]^{1 / 2} / h_{0}^{2}$. Under the condition that electrostatic surface forces dominate the dewetting, $k_{\max }$ $=E_{0}^{2} /(4 \pi \gamma)$ is independent of the film thickness. For dielectric films as those studied elsewhere [14,16], the wave number is closely relevant to the film thickness.

Now let us further consider the interplay of the electric and van der Waals forces in more detail. There are two natural length scales in the system. One is the well-known parameter $a=[|\mathrm{A}| /(2 \pi \gamma)]^{1 / 2}$, which is of the order of one nanometer and appears in the theory of spinodal dewetting by van der Waals force [26]. The other, $b=4 \pi \gamma / E_{0}{ }^{2}$, is directly linked to the relative strength of the surface tension and electrostatic surface forces. Substitution of the above two 
length parameters $a$ and $b$ into (12) yields the characteristic relation: $k_{\max }=\left(1+\left[1+4 a^{2} b^{2} / \mathrm{h}_{0}^{4}\right)\right]^{1 / 2} / 2 b$. Furthermore, here we introduce two dimensionless parameters, $Q=a / b$ and $\xi$ $=h_{0} / a$, to describe the microsystem; thus the dimensionless wave number can be expressed as

$$
k_{0}=h_{0} k_{\max }=\frac{Q \xi\left[1+\sqrt{1+4 /\left(Q^{2} \xi^{4}\right)}\right]}{2} .
$$

Figure 2 shows the variation of the dimensionless wave number versus the dimensionless film thickness . and the system parameter $Q$. It is clearly observed that electrostatic field increases the wave number of the fast growing mode at fixed film thickness. Each characteristic curve in Figure 2 splits the region into two portions, the upper stable portion and the lower instable portion. As an example, consider a thin film system with the typical Hamaker constant $A$ of the order of $10^{-20} \mathrm{~J}$ [26], surface tension $\gamma$ about $0.03 \mathrm{~N} \mathrm{~m}^{-1}$ and film thickness of $0.1 \mu \mathrm{m}$. In the absence of the electric field, the instability wavelength with the fastest growth rate for such a film is estimated as $273 \mu \mathrm{m}$. By introducing a typical weak electrostatic field $E_{0}=0.2 \mathrm{Vmm}^{-1}$, the wavelength with the fastest growth rate is modified as $56.7 \mu \mathrm{m}$. Clearly, external electric field significantly reduces the characteristic wavelength in surface pattern development of thin conductive films. From relations (12) and (13), the wavelength of the dewetting pattern with the fastest growth rate will decrease dramatically with the decrease in the film thickness $h_{0}$ and the increase of electrostatic filed $E$. This indicates a potential application in electrostatic lithography since the electrostatic field is capable of overcoming the limits of laser wavelength in manufacturing increasingly small features for integrated circuits under microns.

\section{Conclusions}

A simple electrohydrodynamic model has been presented for the description of surface instability and relevant surface pattern modulation in a charged thin conductive film. The model indicates that electrostatic surface force causes reduction of the surface pattern wavelength. The model is expected to be useful in understanding a number of phenomena observed in microelectromechanical systems involving charged thin soft films. The present results can be used for controlled surface pattern modulation in a spinodal dewetting scenario via amplification of surface waves of selected modes.

\section{References}

[1] Oron A, Davis S H and Bankoff S G 1997 Rev. Mod. Phys. 69 931

[2] Safran S A and Klein J 1993 J. Physique II (France) 3749

[3] Sharma A 1993 Langmuir 9861

[4] Brochard-Wyart F, DeGennes P-G, Hervert H and Redon C 1994 Langmuir 101566

[5] Sharma A and Reiter G 1996 J. Colloid Interface Sci. 178383

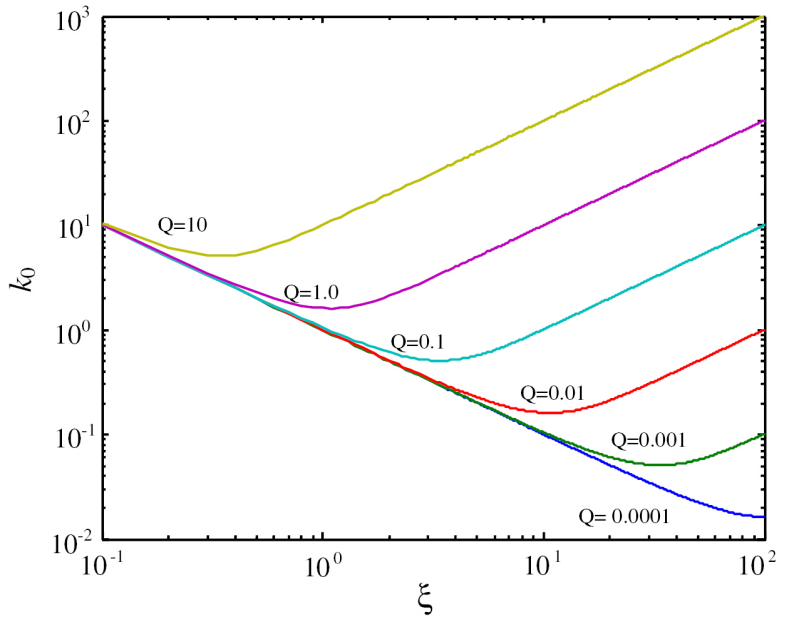

Figure 2. Dimensionless wave number of the fast growing mode versus the dimensionless film thickness and parameter $Q$.

[6] Bischof J, Scherer D, Herminghaus S, and Leiderer P 1996 Phys. Rev. Lett. 771536 [7] Jacobs K, Herminghaus S, and Meche K R 1998 Langmuir 14965

[8] Herminghaus S, Jacobs K, Meche K, Bischof J, Fery A, IbnElhaj M, and Schlagowski S 1998 Science 282916

[9] Shenoy V and Sharma A 2001 Phys. Rev. Lett. 86119

[10] Seemann S, Herminghaus S, and Jacobs K 2001 Phys. Rev. Lett. 865534

[11] Suh K Y and Lee H H 2001 Phys. Rev. Lett. 87135502

[12] Herminghaus S, Seemann R, and Landfester K 2004 Phys. Rev. Lett. 9317801

[13] Warner M R E, Craster R V, and Matar O K 2003 J. Colloid Interface Sci. 268448

[14] Herminghaus S 1999 Phys. Rev. Lett. 832359

[15] Schaffer E, Thurn-Albrecht T, Russell T P, and Steiner U 2000 Nature 403874

[16] Schaffer E, Thurn-Albrecht T, Russell T P, and Steiner U 2001 Europhys. Lett. 53518

[17] Lukatsky D B and Safran S A 2002 Europhys. Lett. 60629

[18] Shankar V and Sharma A 2004 J. Colloid Interface Sci. 274 294

[19] Monch W and Herminghaus S 2001 Europhys. Lett. 53525

[20] Yoo P J, Suh K Y, Park S Y, and Lee H H 2002 Adv. Mater. 141383

[21] Yoo P J, Park S Y, Kwon S J, Suh K Y, and Lee H H 2003 Appl. Phys. Lett. 834444

[22] Yoo P J, Suh K Y, Kang H, and Lee H H 2004 Phys. Rev. Lett. 9334301

[23] Kwon S J, Yoo P J, and Lee H H 2004 Appl. Phys. Lett. 84 4487

[24] Morariu M D, Schaffer E, and Steiner U 2004 Phys. Rev. Lett. 92156102

[25] Landau L D, Lifshitz E M, and Pitaevskii L P 1993 Electrodynamics of Continuous Media 2nd edition (Oxford: Butterworth \& Heinemann)

[26] Israelachvili J 1992 Intermolecular and Surface Forces (New York: Academic) 